BULL. AUSTRAL, MATH, SOC.

VOL. 29 (1984), 423-424.

\title{
COMPOSITE GRAPHS WITH STABILITY INDEX ONE
}

\author{
K.L. McAvaney
}

We characterise the composite graphs with stability index one and those with edge stability index one.

This thesis is motivated by the work of Grant, Holton, and Sims on the semistability of various classes of graphs: an investigation of the relationship between the symmetry of a graph and of its vertex and edge deleted subgraphs. Most of the relevant definitions, results, and background are presented in Chapter 1. A few of the concepts are defined as follows. A graph $G$ is semistable [2] at one of its vertices $v$ if $G$ is trivial or the automorphism group of $G-v$ is the stabiliser of $v$ in $G$. $G$ has stability index one [1] if $G$ is semistable at one or more of its vertices and, for all such vertices $v, G-v$ is not semistable at any vertex. $G$ is pair transitive (introduced as Property $A$ in [3]) if, for every two vertices in $G$, there is an automorphism of $G$ that interchanges them. A composite graph [4] is the cartesian product of two nontrivial graphs. The product is proper [5] if both of the factors contain an edge. Holton and Sims [3] have shown that, with some listed exceptions, a pair transitive composite graph has stability index one. They conjectured a converse for connected composite graphs and thus a characterisation of connected composite graphs with stability index one. The thesis is devoted to the proof of this characterisation and its edge analogue. We also extend the work to disconnected composite graphs.

Received 21 February 1984. Thesis submitted to University of Melbourne, July 1983. Degree approved December 1983. Supervisor; Dr D.A. Holton.

Copyright Clearance Centre, Inc. Serial-fee code: 0004-9727/84 $\$$ A2.00 + 0.00 . 
Chapter 2 deals with the special case of connected singly even graphs: products with an unrepeated 2-path factor. We show that if such a graph has stability index one then it is pair transitive, or it is the product of the 2-path with either the 3-path or the 4-path.

All remaining composite graphs are considered in Chapter 3. We show that if they do not have a prime component then they are pair transitive or the union of one or more copies of one of the singly even exceptions. We find that, with one class of exceptions, all composite graphs with stability index one and a prime component are improper products.

The edge analogue of these results are explored in Chapter 4. We find that a composite graph with no prime component is pair edge transitive if and only if each of its non-trivial components is an $n$-cube for some fixed positive integer $n$. Also we prove that, excluding $n=2$, these are precisely the composite graphs with edge stability index one and no prime component. Finally, we show that all composite graphs with edge stability index one and a prime component are improper products.

\section{References}

[1] D.D. Grant, "The stability index of graphs", Combinatorial Mathematics, 29-52 (Proc. Second Austral. Conf. Combinatorial Mathematics, Melbourne, 1973. Lecture Notes in Mathematics, 403. Springer-Verlag, Berlin, Heidelberg, New York, 1974).

[2] D.A. Holton, "Two applications of semistable graphs", Discrete Math. 4 (1973), 151-158.

[3] D.A. Holton and J.A. Sims, "Graphs with stability index one", J. Austral. Math. Soc. Ser. A 22 (1976), 212-220.

[4] G. Sabidussi, "Graphs with given group and given graph-theoretical properties", Canad. J. Math. 9 (1957), 515-525.

[5] Julie Ann Sims, "Stability of the cartesian product of graphs" (MSc. thesis, University of Melbourne, Melbourne, 1976).

Division of Computing and Mathematics,

Deakin University,

Victoria 3217 ,

Australia. 